\title{
The K-dwarf problem and the time-dependence of gaseous accretion to the Galactic disc
}

\author{
E. Casuso ${ }^{1}$ and J. E. Beckman ${ }^{1,2}$ \\ 1 Instituto de Astrofísica de Canarias, 38200 La Laguna, Tenerife, Spain \\ ${ }^{2}$ Consejo Superior de Investigaciones Científicas, Spain \\ e-mail: jeb@ll.iac.es
}

Received 24 September 2003 / Accepted 19 February 2004

\begin{abstract}
In this article we compare and contrast the predictions of models we have published previously, with the most recent observational data on the G-dwarf/K-dwarf metallicity distribution in the local Galaxy, as well as with up to date plots showing the relation between the $\mathrm{Be}$ and $\mathrm{B}$ abundances of local stars and their $\mathrm{Fe}$ abundances. We show how the data clearly support models in which infall to the plane has not declined secularly during the disc lifetime, and that the best fits are to models in which the accretion rate has tended to a slow increase. These models, devised to give the best possible account of the G-dwarf metallicity distribution, turn out to give an even better account of the K-dwarf distribution. They also do surprisingly well at predicting the observed plots of $\mathrm{Be}$ and $\mathrm{B}$ vs. Fe in the disc.
\end{abstract}

Key words. Galaxy: disk - ISM: evolution - Galaxy: evolution - Galaxy: abundances

\section{Introduction}

Well before ideas about galaxy growth by mergers and accretion became fashionable in the context of cold dark matter $(\mathrm{CDM})$ cosmologies and their inputs on galaxy formation, evidence from chemical evolution in the Galaxy was pointing to the probable infall of gas to the plane during its temporal evolution. Larson (1972a,b) suggested that the growth of the Galactic disc by accretion of low metallicity gas offered a natural explanation for the known dearth of very low metallicity stars, the "G-dwarf problem". An advantage of the infall mechanism over others which invoked for example metal-dependent effects on the initial mass function (IMF), was that it offered an explanation for the deuterium observed close to the Galactic center (Audouze et al. 1976). For a time the most popular scenario for the accretion of gas to the plane of the Milky Way was the effect of the Galaxy in sweeping up material as it passed through the Local Group. Predicted infall rates of a few solar masses per year were inferred for the disc (Hunt \& Sciama 1972; Tinsley 1977), consistent with the observed star formation rate (SFR). Larson 1976 postulated that the infalling matter might be late arrival of gas from the outer parts of the condensing protogalactic nebula. In 1963 Muller et al. discovered the High Velocity Clouds (HVCs) of neutral atomic hydrogen, whose velocities differ from the Galactic rotation velocity by up to several hundred kilometres per second. Now there is extensive observational material on this topic (see e.g. Wakker \& van Woerden 1997, and references therein). Oort (1966) suggested that the HVCs are infalling primordial gas and associated the HVCs

Send offprint requests to: E. Casuso, e-mail: eca@ll.iac.es detected near the Galaxy with the Local Group (Verschuur 1969; Oort 1970). Although the case for the primordiality of the gas is no longer convincing (Lehner et al. 2001; Weiner et al. 1999; Wakker et al. 1999c; Gibson et al. 1999; Lubowich et al. 2000; Wakker 2001; Wakker et al. 2003; Savage et al. 2003; Sembach et al. 2003; Tripp et al. 2003), the fact that at least some HVCs are Local Group members rather than gravitationally bound to the Galaxy has received considerable observational and theoretical support (Mirabel 1982; Songaila et al. 1988; Lepine \& Duvert 1994; Blitz et al. 1999; Lopez-Corredoira et al. 1999; Sembach et al. 1999; Gibson et al. 2001; Casuso \& Beckman 2001; Putman et al. 2002).

There is considerable support for infall as an essential element of chemical evolution of galaxies. For the observations see especially Phookun et al. (1993), Meyer et al. (1994). For theoretical models and scenarios using infall see Cox \& Smith (1976), Hedrick \& Cox (1977), Mayor \& Vigroux (1981), Pryor \& Lear (1983), Clayton (1984), Bothun (1985), Tosi (1988), Ryden (1988), Casuso \& Beckman (1989, 1997, 1999, 2000, 2001), Casuso et al. (1989, 1990), Josey \& Tayler (1991), Olivier et al. (1991), Casuso (1991), Josey \& Arimoto (1992), Toth \& Ostriker (1992), Phookun et al. (1993), Phillipps (1993), Pitts \& Tayler (1996), Pilyugin \& Edmunds (1996), Chiappini et al. (1997), Bouwens et al. (1997), Tantalo et al. (1998), Thon \& Meusinger (1998), Jiang \& Binney (1999), Takeuchi \& Hirashita (2000), Casuso \& Beckman (2003).

In 1978 Reeves \& Meyer suggested that the distribution of the $\mathrm{Be} / \mathrm{Fe}$ ratios for Galactic disc stars could be explained using the infall hypothesis which we will examine in some detail below. 
The evidence from deuterium is particularly interesting. In the solar neighbourhood interstellar medium (ISM) D/H ratios of $1.5 \times 10^{-5}$, first well measured with HST (see e.g. Piskunov et al. 1997), have been more recently confirmed by results from FUSE (Hebrard et al. 2002; Wood et al. 2002; Lehner et al. 2002; Lemoine et al. 2002; Sonneborn et al. 2002; Friedman et al. 2002; Kruk et al. 2002; Moos et al. 2002). In the Galactic center region the $\mathrm{D} / \mathrm{H}$ ratio is an order of magnitude less, as first estimated at $\mathrm{mm}$. wavelengths from deuterated molecules by Jacq et al. (1999), confirmed from radio observations of HCN and DCN by Lubowich et al. (2000), and from ISO-FIR ratios of $\mathrm{HD} / \mathrm{H}_{2}$ by Polehampton et al. (2002). Both the local and the Galactic center D abundances imply continuing infall to the Galaxy of gas which has suffered little previous net astration, the difference between them being due to the much more rapid destruction rate of $\mathrm{D}$ by astration in the Galactic centre. Without continuing infall there would be no measurable D near the Galactic centre, and the local ISM abundance would be a much lower fraction of the estimated primordial abundance which is around twice the local ISM value (see e.g. Pettini \& Bowen 2001).

The strongest indication of infall is found in the now classical "G-dwarf problem" (van den Bergh 1962; Schmidt 1963; Pagel \& Patchett 1975; Tinsley 1980; Pagel 1987). It is notable that there are far fewer low-metallicity stars in the solar neighbourhood than predicted from simple closed-box models for the chemical evolution of the Galactic disc. Integrated light studies imply that this problem is not restricted to the Galaxy (Worthey et al. 1996) but is found in other galaxies similar to the Milky Way (Espana \& Worthey 2002; Bellazzini et al. 2003). As the main sequence lifetimes of late $F$ and $G$ stars are of the order of the present age of the Galaxy, these stars provide a fairly complete record of its evolutionary history. However, even G-dwarfs are sufficiently massive that they have begun to evolve away from the main sequence, and evolutionary corrections must be used when determining their space densities and metallicities. It has long been recognized that K-dwarfs would make a cleaner sample of the local metal abundance distribution, since for these stars the evolutionary corrections are very small. The intrinsic faintness of the K-dwarfs has meant that only recently, however, has accurate spectrometric abundance analysis become available, with which to calibrate photometric abundance indicators (Flynn \& Morell 1997). After excluding local halo stars on kinematic criteria, the statistics of disc stars in a frequency metallicity plot show very low numbers for low disc metallicity, $([\mathrm{Fe} / \mathrm{H}] \leq-1)$, rising sharply to quite a narrow peak between $[\mathrm{Fe} / \mathrm{H}] \simeq-0.6$ and $[\mathrm{Fe} / \mathrm{H}] \simeq-0.2$, and then falling off to higher metallicities. These trends were first measured for the G-dwarfs (Carney et al. 1990; Rocha-Pinto \& Maciel 1996); and occur naturally in a scenario with infall of low-metallicity gas to the Galactic plane (Casuso \& Beckman 1997). The K-dwarfs are now found to exhibit the same "problem": a dearth of K-dwarfs at low disc metallicity. We note here that the K-dwarfs should allow a more accurate record of the chemical history of the Galactic disc (Flynn \& Morell 1997; Favata et al. 1997; Kotoneva et al. 2002).

The second piece of chemical evidence requiring lowmetallicity infall is the evolution of Be and B in the local stellar population. For nearly a decade a large compilation of observations showing that there is a "loop back" in the plot of the Be abundance vs. metallicity in the $[\mathrm{Fe} / \mathrm{H}]$ range between -1 and 0 has been available. An updated version is presented in Fig. 6. It is interesting that most of those presenting chemical models designed either primarily or incidentally to explain light element abundance evolution have treated the disc data very lightly (Vangioni-Flam et al. 1990; Prantzos et al. 1993; Alibes et al. 2002; Valle et al. 2002). One reason is that much attention has been paid to explaining the quasi-linear Be vs. Fe curve at halo metallicities, i.e. for $[\mathrm{Fe} / \mathrm{H}] \leq-1$, and the detailed treatment of Be evolution in the disc has been considered of less interest. It is particularly easy to neglect details of the disc evolution of $\mathrm{Be}$ and $\mathrm{B}$ in the type of $\log$-log representation normally used.

The fact that the Fe metallicity has been approximately constant during the past $5 \mathrm{Gyr}$ is a valid deduction from the best estimates of metallicity vs. age for a wide variety of stellar sources in the solar vicinity (Twarog 1986; Meusinger et al. 1991; Edvardsson et al. 1993; Feltzing et al. 2001). This, together with the relatively high SFR during the same period (Barry 1988; Rocha-Pinto et al. 2000) necessarily implies the arrival of low metallicity gas to counteract the production of $\mathrm{Fe}$ in stars.

We can tie much observational evidence together to give estimates of the long-term SFR based on different methods. Chemical evolution models of the solar cylinder which account well for the evolution with time of the most commonly observed metals: notably iron metallicity and the oxygen/iron ratio (using data for the latter from Nissen et al. 1994; Rebolo et al. 1995; Israelian et al. 1998; Garcia-Lopez et al. 2001) and also for that of the light elements (Casuso \& Beckman 1997, 1999, 2000, 2003), imply an average accretion rate for the Galaxy of $\sim 2 M_{\odot} \mathrm{yr}^{-1}$. Estimates of SNII rates in the Galaxy, of order a few per century in the disc (Dragicevich et al. 1999), are consistent with an arrival rate of gas of a few $M_{\odot} \mathrm{yr}^{-1}$, assuming a classical Salpeter IMF, with mass limits from $0.1 M_{\odot}$ to $100 M_{\odot}$, and assuming that type II SNe are produced by stars with masses $\geq 8 M_{\odot}$. These predictions are in fair agreement with the best estimates of the infall rate due to the HVCs.

The extensive $21 \mathrm{~cm}$ studies by Wakker \& van Woerden (1991), Wakker (1991), Wakker \& Schwarz (1991) and Wakker et al. (1999a,b) on HVCs allow us to make estimates of the current mass flow towards the disc, by estimating the product of the velocity component of a cloud perpendicular to the Galactic plane, its estimated mass, and its estimated distance. Integrating over all clouds deemed to be accreting from outside the Galaxy, inflow rates of between $0.5 M_{\odot} \mathrm{yr}^{-1}$ and $5 M_{\odot} \mathrm{yr}^{-1}$ are derived. The major uncertainty comes from the difficulty of estimating distance. Within these error limits the rates of supply and demand can be balanced in a model within which intergalactic gas, notably the HVCs supplies the metal-poor inflow to the disc.

Another pointer to an extragalactic origin are the low metallicities. Wakker et al. (1999a,b) used sulfur abundance measurements to estimate the metallicity of a specific HVC at 0.094 of the solar value. This is incompatible with a Galactic origin, since the timescales of clouds expelled from the disc and falling 
back are short enough to imply that they would have near solar metallicity. A final argument suggesting that the HVCs are extragalactic is that their properties are essentially similar to those of the Lyman alpha forest clouds (Blitz et al. 1999).

Lopez-Corredoira et al. (1999) used the constraint that the inflow rate of the HVCs should have remained essentially constant over the lifetime of the Galactic disc to make an estimate of the implied total mass of the HVCs within the Local Group, finding that some $50 \%$ of the Local Group mass should be in HVCs (they used a prescription for an HVC in which some $20 \%$ is in baryons, and the rest in cold dark matter). This is in fair agreement with dynamical estimates of the Local Group mass compared with the masses of its known component galaxies.

The wealth of articles dealing with light element production and evolution during the Galaxy lifetime, in halo and disc, can barely be touched on here. Agreement between model predictions and observations has not been particularly good. Although reasonable fits to the near linear dependence of Be and $\mathrm{B}$ on $\mathrm{Fe}$ or $\mathrm{O}$ for halo stars have been produced (Ramaty et al. 2000; Fields et al. 2000), this cannot be said for the disc. Even the most recent and most complete (see e.g. Valle et al. 2002) models, which apply a full range of possible nuclear reactions: spallation between $\mathrm{C}, \mathrm{N}, \mathrm{O}$ nuclei and protons, and alpha particles, as well as alpha+alpha fusion, and include refinements such as quantifying different trapped fractions, and an explicit treatment of the time evolution of the intensity of the Galactic cosmic rays (GCR's) do not come very close to reproducing the observed plots of $\mathrm{Li}, \mathrm{Be}$ and $\mathrm{B}$ vs. Fe (or O) in the disc. The key features not well reproduced are the steep slope of the relation for $\mathrm{Li}$, and the loopback, i.e. non-monotonic relation for Be and B, (see Casuso \& Beckman 1997, 2000). This is mainly because the modelers have not paid sufficient attention to the effects of gaseous infall, which modulate all the abundance relations, when combined with the different production and (in the case of $\mathrm{Li}$ ) destruction mechanisms which operate. In the following section we will show how infall models can account in general for the observations, and that a specific infall model which we have presented previously not only explained well the observations available prior to its publication, but gives even better fits when data published subsequently to its publication are taken into account.

\section{Comparison of predictions and observations}

\subsection{Metallicity histograms}

The range of models which we are relying on here to explain the observed abundances, both in stars and in the ISM, have been treated in detail in a series of previous articles (Casuso \& Beckman 1997, 1999, 2000, 2001, 2003), so we will not give further details here. They make use of well established formalism for treating the production of light element nuclides by GCR protons and alpha particles in the ISM, together with a classical chemical evolution model which predicts the abundances of heavier elements in the ISM as a function of epoch. The only significantly individual inputs are the incorporation of gaseous infall at a time averaged rate which is either constant

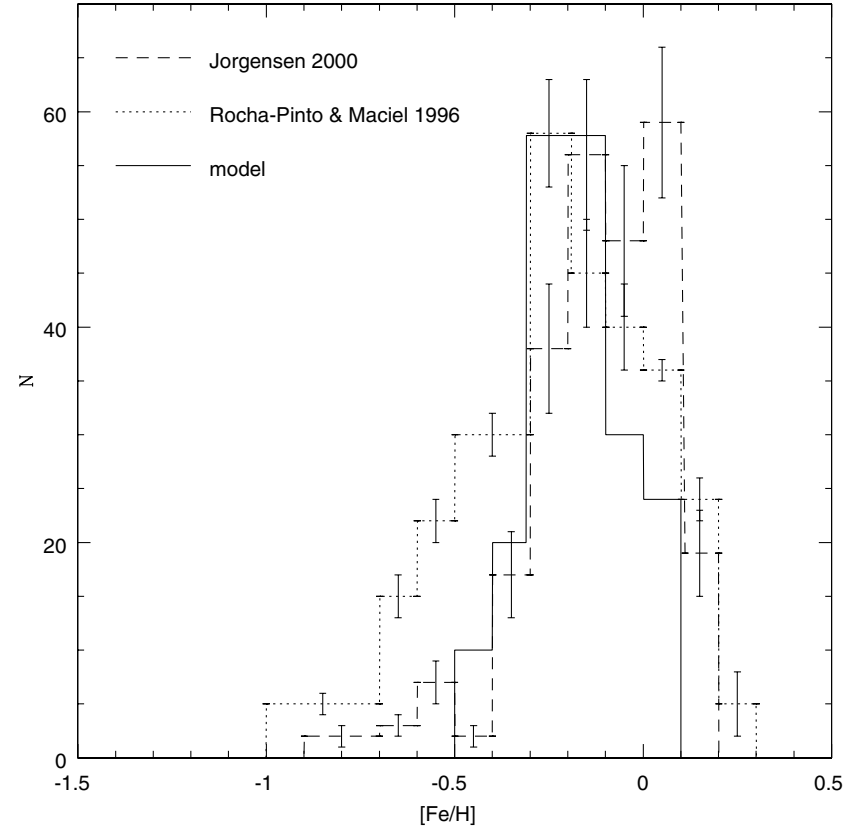

Fig. 1. Metallicity distribution function of disc stars. Our increasing infall model prediction (solid histogram) was made in Casuso \& Beckman (2001) to explain the main peak observed by Rocha-Pinto \& Maciel's (1996) (dotted histogram). The sharp increase near $[\mathrm{Fe} / \mathrm{H}]=$ -0.3 implied by our theoretical prediction yields a rather narrow global distribution in comparison with Rocha-Pinto \& Maciel's data.We attribute this to the presence of thick disc stars in their data set. However the prediction is better supported by the more recent data (dashed histogram) of Jorgensen (2000). To be conservative we adopt an $[\mathrm{Fe} / \mathrm{H}]$ error of between 0.1 and $0.2 \mathrm{dex}$.

or steadily increasing with epoch, plus the effects of stochastic variations in the infall rate on shorter timescales due to the partially discretized arrival rate (i.e. as clouds) of intergalactic gas.

In Fig. 1 we show observational data from Rocha-Pinto \& Maciel (1996) on the G-dwarf metallicity distribution in the local Galactic disc. We compare this with the prediction of a model published by Casuso \& Beckman in (2001), using the hypothesis of slowly increasing infall of low-metallicity gas from the intergalactic medium, aimed at explaining these data. We can see that the model predicts a peak with a considerably narrower metallicity distribution than these observations. In the same figure we have included a more recent compilation for G-dwarfs by Jorgensen (2000), published while Casuso \& Beckman (2001) was in press. The new observations show a much narrower peak than the previous set, in improved agreement with our predictions. Looking back for similar studies which we had not included in our 2001 paper we found a data set for local G- and F-dwarfs published by Wise \& Gilmore, which, though giving a slightly wider frequency distribution than our model prediction, is also in fair agreement with it; we show this in Fig. 2.

In Fig. 4 we show how recent measurements using K-dwarfs compare with our model predictions. Figure 3 shows metallicity distribution function of disc stars (MDF) using $\mathrm{Fe}$ as the metallicity indicator, from Favata et al. (1997). 


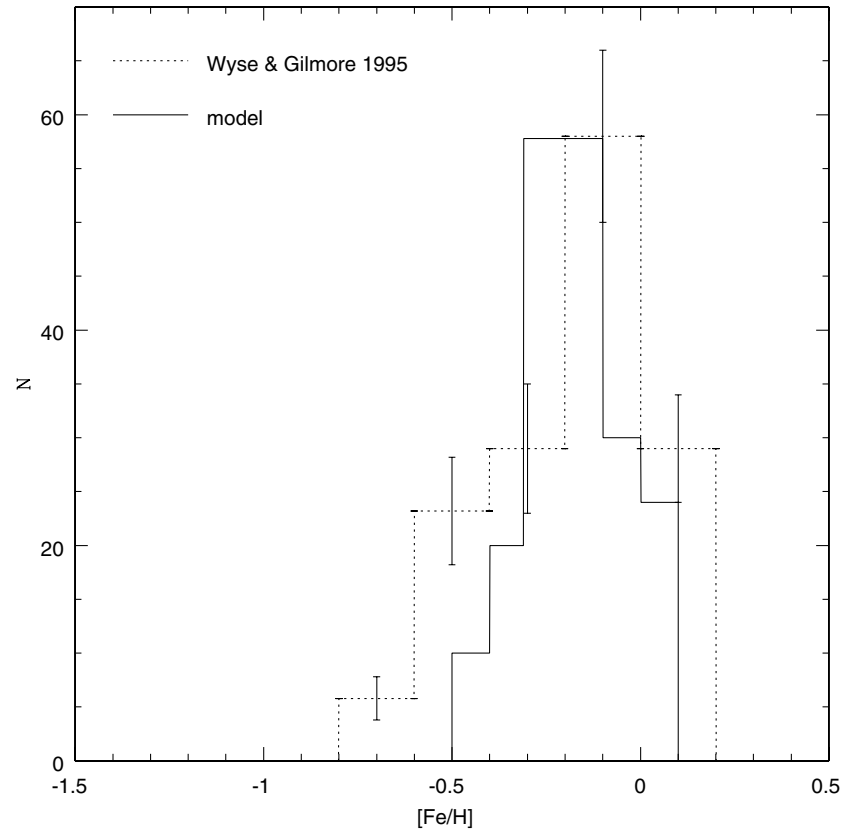

Fig. 2. Metallicity distribution function of disc stars. The output of our infall model (solid histogram). Data are from Wyse \& Gilmore (1995) for $\mathrm{F} / \mathrm{G}$ disc stars (dotted histogram). To be conservative we adopt an $[\mathrm{Fe} / \mathrm{H}]$ error of between 0.1 and $0.2 \mathrm{dex}$.

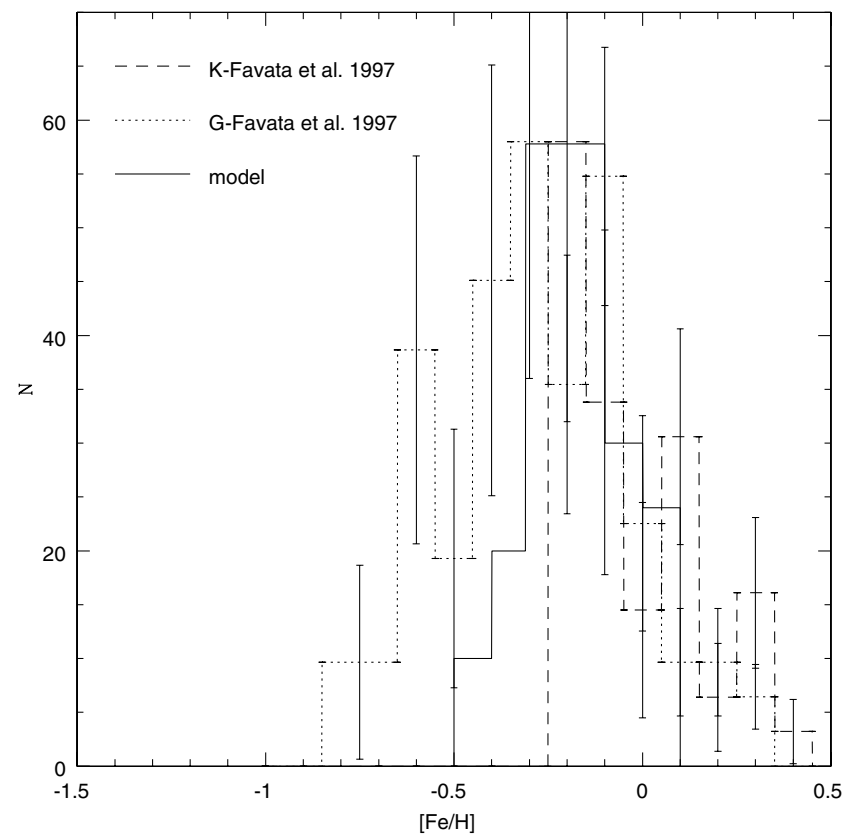

Fig. 3. Metallicity distribution function of disc stars. For comparison, we present here data from Favata et al. (1997) for G-dwarfs (dotted histogram), and from Favata et al. (1997) for K-dwarfs (dashed histogram). The output of our increasing infall model (solid histogram). To be conservative we adopt an $[\mathrm{Fe} / \mathrm{H}]$ error of between 0.1 and 0.2 dex.

They observed sets of G-dwarfs and also K-dwarfs. In the figure we match their frequency plots with the output of our best infall model from Casuso \& Beckman (2001). The key point here is that the K-dwarf peak centered on $[\mathrm{Fe} / \mathrm{H}] \simeq-0.25$ is considerably narrower than that for the G-dwarfs. We would

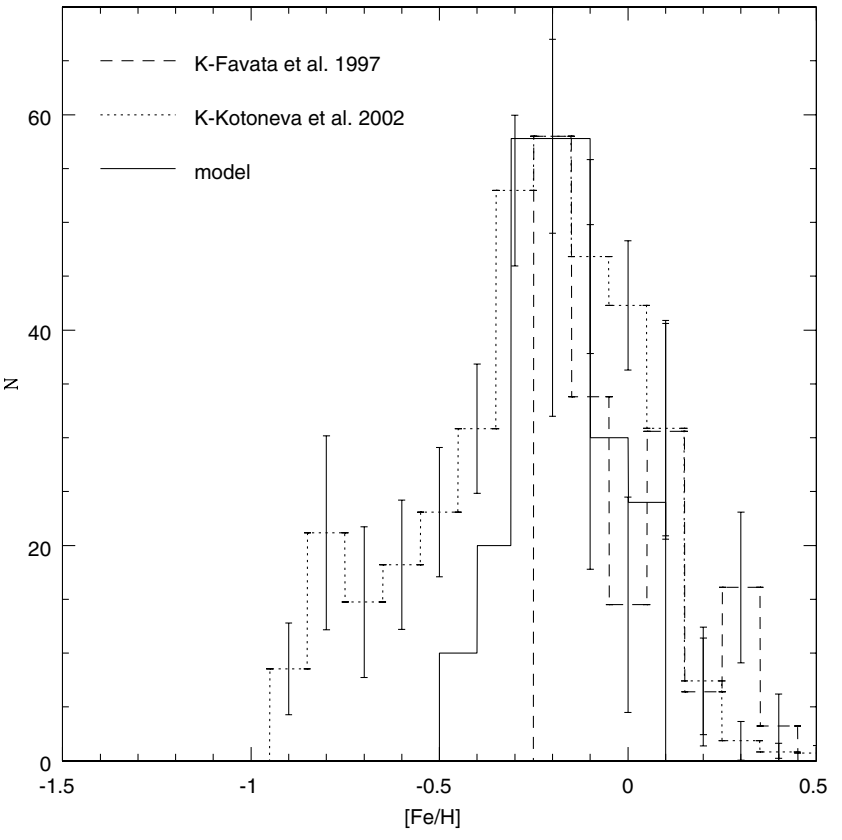

Fig. 4. Metallicity distribution function of disc stars. The output of our infall model (solid histogram). Data from Kotoneva et al. (2002) for K-dwarfs are presented for comparison (dotted histogram), and from Favata et al. (1997) also for K-dwarfs (dashed histogram). To be conservative we adopt an $[\mathrm{Fe} / \mathrm{H}]$ error of between 0.1 and 0.2 dex.

expect that the K-dwarfs are significantly less affected by evolutionary effects, and as such are a better reflection of the main sequence distribution. The fact that the K-dwarf distribution is better approximated by our model prediction, as seen in Figs. 3 and 4 , is what we would have expected from the assumptions of the model, which does not set out to model stars as they evolve away from the main sequence. In Fig. 4 we compare, again, our predicted distribution with recent observations of the K-dwarf $\mathrm{Fe} / \mathrm{H}$ frequency distribution from observations by Kotoneva et al. (2002). One point to make when confronting theory with observation here is the scatter in the observational results; two observational plots presented in Figs. 3, 4 differ above all in the widths of the tails in their distributions, though they are in good agreement about the metallicity value, and the half width, of the peak.

Since we have been comparing the observations with only one model, it is instructive now to show a similar comparison with other models which take different basic assumptions (see Fig. 5). There are a wide variety of these, and those we have chosen are generically representative rather than comprehensive. Firstly we have taken two closed box models, one with the instantaneous recycling approximation (see e.g. Tinsley 1980; Pagel 1987), and the other where this constraint has been relaxed. We can see clearly that these models predict too many low metallicity stars, and this well known result does not need further comment here. Secondly we have taken a recent model with gaseous infall, from Kotoneva et al. (2002) which uses the standard assumption that this infall must be declining with time, and has taken an exponential decline as an analytical approximation, which again is a commonly used assumption (Lynden-Bell 1975; Clayton 1984, 1988). We can see that even 


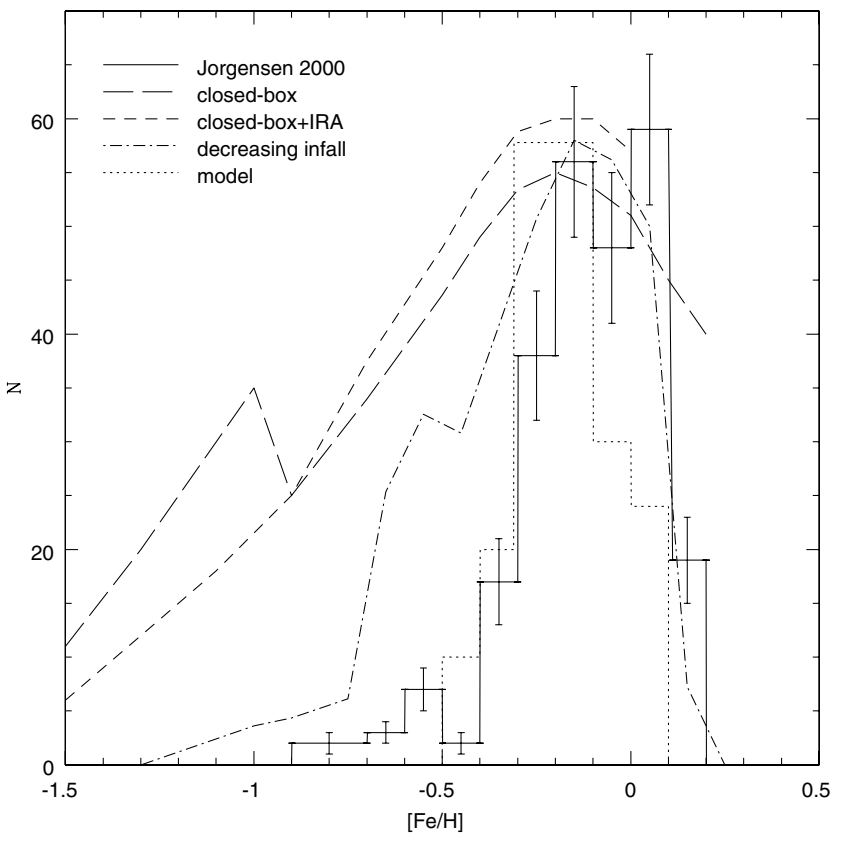

Fig. 5. Metallicity distribution function of disc stars. Data are from Jorgensen 2000 (solid histogram). We show, for comparison, the predictions of: simple closed box model using instantaneous recycling approximation (IRA) (short dashed line), the same model without IRA (long dashed line), a model using infall exponentially decreasing with time (from Kotoneva et al. 2002) (dotted-dashed line), and our prediction using increasing infall (dotted histogram).To be conservative we adopt an $[\mathrm{Fe} / \mathrm{H}]$ error of between 0.1 and 0.2 dex.

this model is predicting too many stars with metallicities between -1 and -0.5 ; its peak is considerably narrower than the broad peaks for the closed box models, but it is not a really good approximation to the data, certainly not compared to a model with increasing infall rate, of the kind we have used in the previous figures. The model of Kotoneva et al. is for this purpose typical of these types of models; we have tested others which make the same assumption, and they all predict very similar results. Since an increasing infall rate is also the best way of explaining the time dependence of the SFR as measured using the entirely different technique of chromospheric indicators, (Rocha-Pinto et al. 2000), we claim that the data present strong evidence for secularly increasing infall.

On comparing the observations with the model output in Figs. $1-3$, and above all in Fig. 4 we can immediately see an excess, well outside any effects due to observational error, in the observed stellar frequency and the predicted frequency in the range $-1 \leq[\mathrm{Fe} / \mathrm{H}] \leq-0.5$. The reason for this excess is that our model handles only the conditions of the long timescale stellar formation (of order $10 \mathrm{Gyr}$ ) which pertain to the thin disc of the Galaxy. In the range of metallicity specified, however, there is a measurable contribution from the "thick disc" which formed on a timescale of order 1 to $1.5 \mathrm{Gyr}$ from the initiation of the proto-Galactic collapse (Mashonkina et al. 2003), and was an intermediate phase between the halo and the thin disc (see e.g. Pardi et al. 1995). The thick disc is now an observationally well established feature of the Galaxy, whose

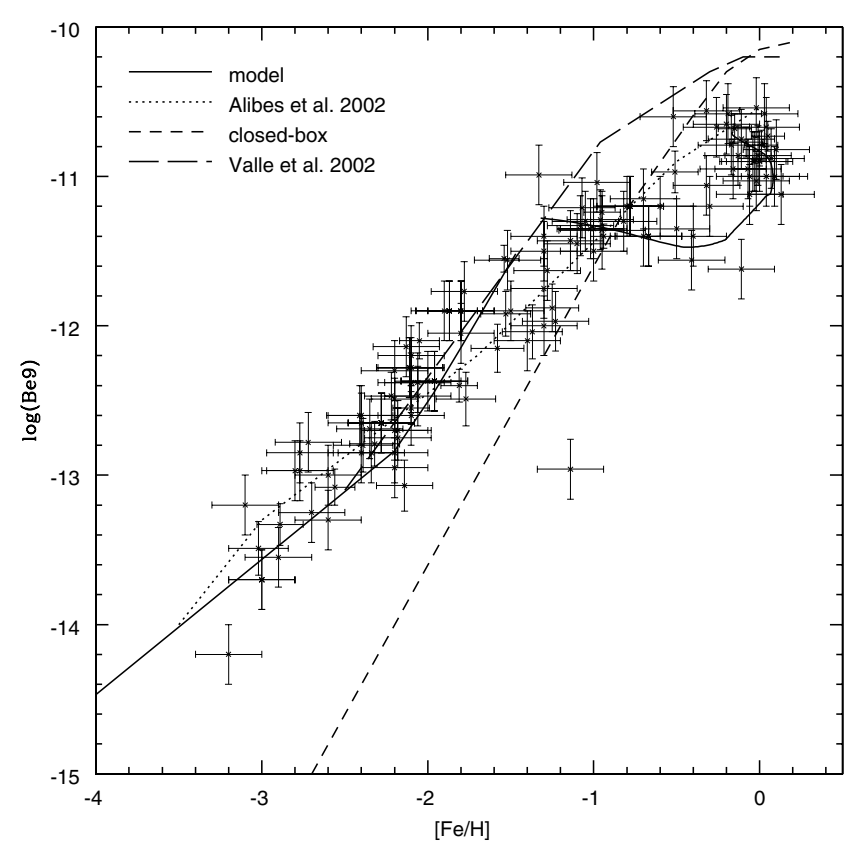

Fig. 6. Our model (halo and disc separated) production of Be as a function of $[\mathrm{Fe} / \mathrm{H}]$ (full line). For comparison we show the theoretical predictions of: Alibes et al. (2002) (dotted line) who use a model with exponentially decreasing infall, Valle et al. (2002) who use two models: a) without external infall but a multizone model in which gas suffers radial interchange (long-dashed line), and b) closed-box model (dashed line). Data from Rebolo et al. (1995), Gilmore et al. (1992), Nissen et al. (1994), Ryan et al. (1992), Boesgaard \& King (1993), Grevesse \& Sauval (1998), Chmielewski et al. (1975), Boesgaard et al. (1999), Primas et al. (2000a,b).

MDF peaks at $[\mathrm{Fe} / \mathrm{H}] \sim-0.7$ (Gilmore et al. 1995). There is as yet no generally agreed canonical model for the formation of this feature, however. Among the possibilities which have been proposed are the puffing up of the disc by galaxy harassment (Bienayme 1999), a specific violent merger event or a close encounter with a companion galaxy (Robin et al. 1996; Bensby et al. 2003; Feltzing et al. 2003). Measurements of the abundances of Fe, Ca, Si, Ti, Co, V, Eu, Mg, Mn, Al, and $\mathrm{Zn}$, have demonstrated that the thick disc has a genuinely different chemical history, which differs from that of the thin disc (Naoumov 1999; Prochaska et al. 2000). However there is a significant metallicity overlap between the two disc populations, as measured via $\mathrm{Fe}$, in the range $-1 \leq[\mathrm{Fe} / \mathrm{H}] \leq-0.1$ (Wyse \& Gilmore 1995). The presence of lenticular as well as the "standard" bulge and disc components in spiral galaxies has been well known and seriously studied purely morphologically since the classical paper by Kormendy (1979). The presence of well defined thick discs has recently been established in galaxies other than the Milky Way (Sarajedini \& van Duyne 2001; Dalcanton \& Bernstein 2002).

\subsection{Berillium versus iron in the disc}

In Fig. 6 we show a comparison of model predictions for the evolution of $\mathrm{Be}$ (measured against $\mathrm{Fe}$ ) in the disc with a major compilation of observed Be abundances in solar nighbourhood 
stars of both disc and halo. The sources of the data are given in the figure caption, and represent a significant up-date on those available for a previous compilation we reported in Casuso \& Beckman (2001). We can immediately see in Fig. 6 that there is a clear dichotomy between the disc behaviour and that of the halo, so we should state clearly here that although we have previously produced a halo model (Casuso \& Beckman 1997), in which gas flow plays a key role in producing the quasi-linear relation between $\mathrm{Be}$ and $\mathrm{Fe}$, we are concentrating only on the disc in the present paper.

A virtue of logarithmic abundance plots of the type used in Fig. 6 is that they do justice to evolution during phases of low abundance. For this reason Be evolution in the halo is especially well displayed in Fig. 6. However as a corollary they do less than justice to phases of high abundance. The part of the graph which refers to the disc appears squeezed into one corner, even though in absolute terms some $90 \%$ of the current Be was produced in the disc. This, plus the fact that the linear plot of $\mathrm{Be}$ vs. $\mathrm{Fe}$ in the halo was a real challenge to theorists, and could have had direct repercussions on certain Big Bang nucleosynthesis predictions, has led to a rather cavalier treatment of the disc data by modellers. In Fig. 6 we show three examples of not very impressive fits to the observations. As just explained, it would not be reasonable to criticize the authors of these models strongly for this, since their goals were directed at the halo and since their fits to the halo data are good.

The main aims of halo modelers (Fields et al. 2000; Ramaty et al. 2000; Alibes et al. 2002; Valle et al. 2002) is to elucidate the nuclear processes involved in the production of Be (also B) rather than to produce chemical evolution models, so they used standard assumptions, above all for the disc evolution. The fact that in a log-log plot the disc appears as an appendage of the halo aided them in not needing to pay special attention to the disc. The only point we wish to make here is that they did use a standard infall assumption for the disc, i.e. infall declining with epoch, with the results shown in Fig. 6. As well as obtaining a good fit to the halo data, they obtain a plot which passes close to regions with many disc data points, but this does not mean that they fit the observed trend, as can be clearly seen in the figure. Valle et al. (2002) do not call on external infall in their models. One of these, a closed-box model, fails to fit either halo or disc observations, but this is just an example of how closedbox models do not account for chemical evolution observations in general, and the authors clearly show this model as a comparison. In the other model type, in which metal interchange between different radial zones of the Galaxy is used, but without recourse to external infall as such, the fit to the halo data is fair, but to the disc data is not very good. The other model included in this figure is the increasing exponential infall model of Casuso \& Beckman (1997) a model to be applied to the disc only (as explained above, the halo was modeled using different assumptions, relevant to the epoch of its formation). In this model the predicted plot of Be vs. Fe shows a loop-back, and offers an excellent fit to the observations. It would be natural to suspect that the model was engineered to fit the observations in this way, and this suspicion is fully justified. However that is what a model is supposed to be designed for. This fit might even be used by some dismiss the model were it not for the fact

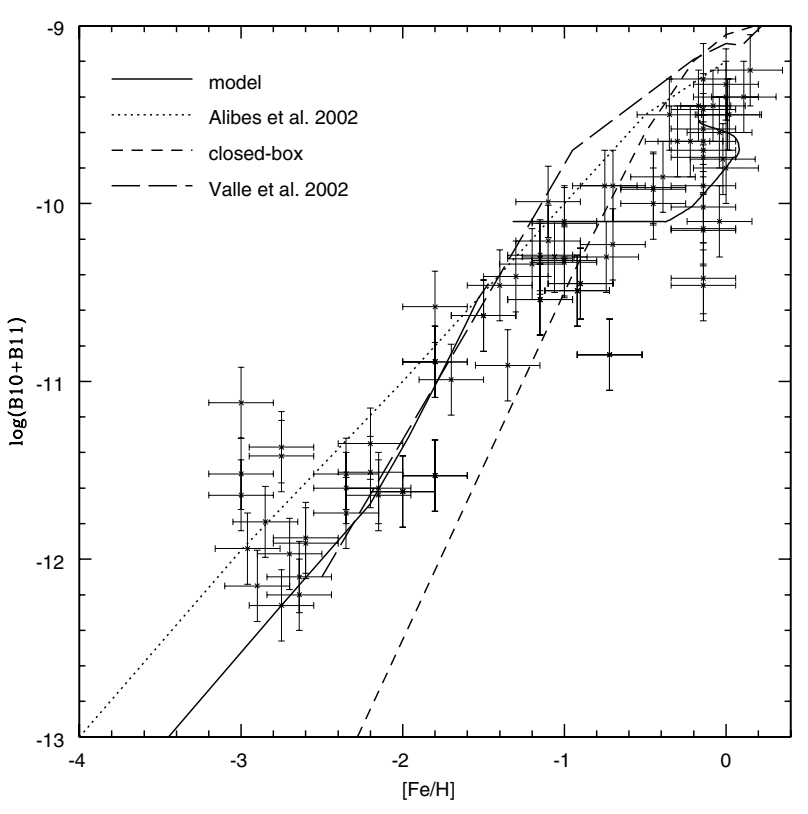

Fig. 7. Our model production of ${ }^{11} \mathrm{~B}+{ }^{10} \mathrm{~B}$ as a function of $[\mathrm{Fe} / \mathrm{H}]$ (full line). For comparison we show the theoretical predictions of: Alibes et al. (2002) (dotted line) who use a model with exponentially decreasing infall, Valle et al. (2002) who use two models: a) without external infall but a multizone model in which gas suffers radial interchange (long-dashed line), and b) closed-box model (dashed line). Data from the same authors as in Casuso \& Beckman (1997) but added are data from Grevesse \& Sauval (1998), Howk et al. (2000), Cunha et al. (2000), Primas et al. (1999), Duncan (1997), Garcia-Lopez et al. (1998). We have taken an average between LTE and NLTE values reported by the authors, including this effect in the error bars. Solid lines represent the prediction of our model, which is divided into halo and disc models, with differing assumptions (see Casuso \& Beckman 1997).

that it makes better predictions for the G- and K-dwarf metallicity distributions than competing models.

\subsection{Boron versus iron in the disc}

In Casuso \& Beckman (1997) we give a plot of the data on the abundance of boron in local Galactic halo and disc stars, from the state of the observations in 1997, overlaid with a plot of the prediction from our Galactic infall model published in Casuso \& Beckman (1997). The same criteria apply as for the $\mathrm{Be}$, in the sense that the halo observations and model, for the range of $[\mathrm{Fe} / \mathrm{H}] \leq-1$, are to be treated separately and will not be discussed further here. As for the disc, although the model prediction "fitted" the 1997 data compilation, the number of points was clearly too few to either confirm the model or rule out competing models. In Fig. 7 we reproduce exactly the same model prediction, without any attempt at tuning, and now also include all the observations which have been obtained since then. The number of data points is still less than half those available for $\mathrm{Be}$, because all the observations are from spacebased telescopes, so the statistics are not as good. Nevertheless we can see that the infall model with an exponential slow increase gives a fair account of the observations, and in any case a strikingly better account than other model predictions, of which 
examples are given in Fig. 7. One of these is a closed-box model, which one would not expect to give a close fit to the data. The others are an exponentially declining infall model, and a model in which gas mixing between different zones of the Galaxy is the dominating effect.

\section{Summary}

The model using exponentially decreasing infall does appear to fit better some of the recent MDFs: that of Kotoneva et al. for K-dwarfs (Fig. 4), that of Favata for G-dwarfs (Fig. 3), and that of Rocha-Pinto and Maciel (Fig. 1). The principal problem with this model is that it implies quasi-linear behaviour for the Be vs. Fe and for B vs. Fe (Figs. 6 and 7) in contrast to the observational data. Our models with increasing infall give better fits to the Jorgensen data on G-dwarfs (Fig. 5), and also those of Wyse \& Gilmore (Fig. 2) as well as the Favata et al. MDF for K-dwarfs (Fig. 3). It is of particular interest to note that Jorgensen used a weighting scheme for his G-dwarf data which favoured the stellar population closer to the galactic plane, and therefore selectively tended to eliminate the thick disc population, an important point which we will pick up again later. The model with increasing inflow also gives a very good account of the disc evolution of $\mathrm{Be}$ and $\mathrm{B}$ with $\mathrm{Fe}$ (Figs. 6 and 7). The global hypothesis invoking a slowly increasing rather than a declining infall rate gives good fits to both the MDFs and the Be/B vs. Fe observations, in whichever detailed form we use it. In our previous papers we first (Casuso \& Beckman 1997) presented a simple model with a monotonic exponential decline, and more recently (Casuso \& Beckman 2001) a more complex model in which we took into account the interaction of the infalling gas with the in plane gas, in order to give a better description of the data set on the stellar age distribution, i.e. the history of the local SFR, by Rocha-Pinto et al. (2000). However the differences in the predicted MDFs and the $\mathrm{Be} / \mathrm{B}$ vs. Fe plots for these two models are not significant; in other words the increasing infall assumption dominates the predictions for these parameters, and gives superior results for them than do its rivals, and in addition only models based on this assumption give a decent account of the local SFR history.

It is true that models of the growth of galaxies in cosmological simulations of large scale structure formation, in their current state of development, predict a global decline in the infall of gas both to galaxy groups and to individual galaxies, but here it is necessary to point out that these computations do not have the resolution to take into account in a satisfactory way the effects of the low mass tail of the galaxy mass function. This takes the form of major gas clouds without star formation which remain bound into the groups of galaxies, (Knight \& Ponman 1997; Murali et al. 2002) and which in small galaxy groups, or in the outskirts of larger clusters, are absorbed into the larger galaxies on very extended timescales.

Although infall as a component of chemical evolution models of the Galaxy has become non-controversial, it is of considerable interest to understand it in greater detail, because it can now be seen in the wider context of the continuous merger and accretion processes occurring in galaxies in general. As the mean galaxy density has fallen over cosmological timescales it is usually taken for granted that mergers and interactions of all types were much more frequent at high redshifts. It is also an accepted result of observations that the global star formation rate in the universe has declined as a function of time, for similar reasons: the SFR reflects strongly those processes which are active at the time of galaxy formation. However these broad statements omit certain specifics which are clearly of considerable importance, and in particular they do not take enough account of the differences between spheroid and disc formation.

Once a galaxy cluster or gravitationally bound group has separated out, so that its internal gravity dominates locally over the Hubble flow, the mean density of matter in the cluster is no longer falling. The galaxies within the group develop in the long term in a less dramatic way than initially, but the merger and accretion rate need no longer be a declining function of time. As a result of the distributed orbits of the galaxies around the cluster barycenter the initially larger galaxies will tend to accrete their smaller companions. This process will occur for small galaxies and also for "failed galaxies" the gas clouds whose masses are too small in relation with their Jeans mass for gravitational condensation into stars to have taken place. These failed galaxies, which could have masses of up to $10^{8} \mathrm{M}_{\odot}$ in HI, (plus their corresponding proportion of dark matter) and would certainly comprise many objects of lower mass, are the HVCs which have contributed to the build-up of the Galactic disc, in the case we have discussed in this article. The accretion process should be continuous, a steady "rain" of HI onto the disc, punctuated from time to time by the arrival of one of the more massive clouds, which will stimulate star formation in a measurable way. It is interesting to point out that the densities of the HVCs $\left(\simeq 10^{-4} \mathrm{~cm}^{-3}\right)$ and their measured column densities, imply size scales of kpc, so that the infall of one of the more massive of these clouds will affect a major fraction of a Galactic disc, while the continuous infall of small clouds would be affecting the whole disc continuously but in a random way.

There are two corollaries of this scenario which we would like to point out here. As a galaxy accretes mass, and specifically as it accretes gas to its disc, its accretion power tends to rise: its mass grows, and its accretion cross-section also grows. These factors would naturally give rise to an accretion rate which increases with time. However in a given galaxy group the mean density of intracluster gas will be falling as the gas is pulled into the larger galaxies, an effect which will cause the accretion rate to fall. Which of these effects wins out to determine whether the net accretion rate is rising or falling at a given epoch depends on the initial mean mass density in the clouds, compared to the mass distribution of the initial major galaxies. In Lopez-Corredoira et al. (1999) we showed what the implication of this condition would be for the Local Group. We showed that if the intracluster gas represents at least half the total baryonic mass of the Local Group, then the accretion rate to the Milky Way will have tended to increase with time. This condition appears to be satisfied since the dynamically estimated mass of the Group (Kahn \& Woltjer 1959) is more than twice the combined masses of the Milky Way and M31, the total mass of the remaining galaxies being too small to affect the equation. 
The second effect is seen in estimates of the time dependence of the local SFR as measured via chromospheric activity indicators. The most complete data set is that of Rocha-Pinto et al. (2000), and shows a tendency for the SFR to increase on timescales of $5 \mathrm{Gyr}$ and more, but also, on timescales of $1 \mathrm{Gyr}$ and less, strong deviations from uniform behaviour. These "bursts" (they are not powerful in that the SFR does not grow above a few $M_{\odot} \mathrm{yr}^{-1}$ but their net effect integrated over time is very significant) are not trivial to explain. The sample is a local one, from the solar neighbourhood, but at a given epoch it must contain stars from a considerable radial and azimuthal range, several $\mathrm{kpc}$ in all directions, due to orbit mixing and diffusion. This implies that any "local" upsurge in the SFR must really be occurring simultaneously over a significant fraction of the Galactic disc, since if it were really local its effects would be completely diluted by mixing in the observed sample. Resonance effects such as those which give rise to spiral arms will not produce this behaviour, as the disc averaged SFR in a spiral arm system is not varying, even though the local SFR is varying periodically. The accretion of a major HVC, on the other hand, will yield a more generalized increase in the SFR, over kpc scales, as suggested above. Thus the existence of evidence for substantial time variations of the SFR measured locally is clear evidence in favor of the HVC model of infall, in which there are sporadic arrivals of massive clouds in a constant "rain" of less massive clouds.

The graphs of stellar Be and B abundances against a metallicity index such as the Fe abundance for local disc stars are similar, and show far from linear behaviour, which has not really attracted the attention of the modelers, for reasons which have been outlined above. We have shown here that the evolutionary model which best describes the G- and K-dwarf metallicity distributions observed in the disc: in which the infall rate increases slowly rather than declines, also gives a good prediction of the $\mathrm{B}$ and $\mathrm{Be}$ vs. Fe relations. In a previous study we showed that the Li vs. Fe graph for stars in the disc metallicity range $([\mathrm{Fe} / \mathrm{H}] \geq-1)$ is also well explained in the framework of an infall model, though the interplay of production and destruction processes for Li makes it less useful as a diagnostic for distinguishing between models with different time profiles for the infalling gas.

As the hypothesis of increasing infall goes somewhat against the conventional wisdom we feel that it is in order to offer some testable predictions rather than simply fit already existing data sets. The first is that the iron abundance in the local interstellar medium ought to be rather lower than the solar abundance: numerically $[\mathrm{Fe} / \mathrm{H}]_{\mathrm{ISM}} \simeq-0.2$, an effect due to the secular dilution of the metallicity by the infalling gas over the past $\sim 5$ Gyr. The second is the presence of the "high metallicity plateau" for lithium abundances, which strictly speaking had been observed before we produced the increasing infall models (see Casuso \& Beckman 2000), but has since been confirmed by new data with refined analysis by Chen et al. (2001). A third prediction is related to the K-dwarf problem itself. We suggest that for the stellar population at decreasing distances from the Galactic centre the MDF for disc stars should widen to lower metallicities, because there should be a dynamical trend for the HVC material to flow preferentially to the plane in the outer disc of a rotating galaxy, so that the metallicity dilution by infall will be fractionally less as the Galactic centre is approached.

Acknowledgements. We are very happy to thank Dr. C. Flynn for the very helpful comments and suggestions during the refereeing process. This research was supported by grant AYA2001/0435 of the Spanish Ministry of Science and Technology.

\section{References}

Alibes, A., Labay, J., \& Canal, R. 2002, ApJ, 571, 326

Audouze, J., Lequeux, J., Reeves, H., \& Vigroux, L. 1976, ApJ, 208, L51

Barry, D. C. 1988, ApJ, 334, 436

Bellazzini, M., Cacciari, C., Federici, L., Fussi Pecci, F., \& Rich, M. 2003, A\&A, 405, 867

Bensby, T., Feltzing, S., \& Lundstrom, I. 2003, A\&A, 410, 527

Bienayme, O. 1999, Ap\&SS, 265, 225

Blitz, L., Spergel, D. N., Teuben, P. J., Hartmann, D., \& Burton, W. B. 1999, ApJ, 514, 818

Boesgaard, A. M., \& King, J. R. 1993, AJ, 106, 2309

Boesgaard, A. M., Deliyannis, C. P., King, J. R., et al. 1999, AJ, 117, 1549

Bothun, G. D. 1985, AJ, 90, 1982

Bouwens, R. J., Cayon, L., \& Silk, J. 1997, ApJ, 489, L21

Braun, R., \& Burton, W. B. 1999, A\&A, 341, 437

Carney, B. W., Latham, D. W., \& Laird, J. B. 1990, AJ, 99, 572

Casuso, E. 1991, Ph.D. Thesis, Univ. of La Laguna (Spain)

Casuso, E., \& Beckman, J. E. 1989, Ap\&SS, 156, 39

Casuso, E., Beckman, J. E., \& Cepa, J. 1989, Ap\&SS, 157, 89

Casuso, E., Beckman, J. E., \& Cepa, J. 1990, Ap\&SS, 170, 275

Casuso, E., \& Beckman, J. E. 1997, ApJ, 475, 155

Casuso, E., \& Beckman, J. E. 1999, AJ, 118, 1907

Casuso, E., \& Beckman, J. E. 2000, PASP, 112, 942

Casuso, E., \& Beckman, J. E. 2001, ApJ, 557, 681

Casuso, E., \& Beckman, J. E. 2003, PASJ, 55, 247

Chen, Y. Q., Nissen, P. E., Benoni, T., \& Zhao, G. 2001, A\&A, 371, 94

Chiappini, C., Matteucci, F., \& Gratton, R. 1997, ApJ, 477, 765

Chmielewski, Y., Brault, J. W., \& Muler, E. A. 1975, A\&A, 42, 37

Clayton, D. D. 1984, ApJ, 285, 411

Clayton, D. D. 1988, MNRAS, 234, 1

Cox, D. P., \& Smith, B. W. 1976, ApJ, 203, 361

Cunha, K., Smith, V. V., Boesgaard, A. M., \& Lambert, D. L. 2000, ApJ, 530, 939

Dalcanton, J. J., \& Bernstein, R. A. 2002, AJ, 124, 1328

Dragicevich, P. M., Blair, D. G., \& Burman, R. R. 1999, MNRAS, 302, 693

Duncan, D., Lambert, D., \& Lemke, D. 1992, ApJ, 401, 584

Duncan, D., et al. 1995, private communication

Duncan, D., Primas, F., Rebull, L. M., et al. 1997, ApJ, 488, 338

Edvardsson, B., Andersen, J., Gustafsson, B., et al. 1993, A\&A, 275, 101

Espana, A. L., \& Worthey, G. 2002, AAS, 201, 1408

Favata, F., Micela, G., \& Sciortino, S. 1997, A\&A, 323, 809

Feltzing, S., Holmberg, J., \& Hurley, J. R. 2001, A\&A, 377, 911

Feltzing, S., Bensby, T., \& Lundstrom, I. 2003, A\&A, 397, 1

Fields, B. D., Olive, K. A., Vangioni-Flam, E., \& Casse, M. 2000, ApJ, 540,930

Fitzpatrick, E. L. 1996, ApJ, 473, L55

Flynn, C., \& Morell, O. 1997, MNRAS, 286, 617 
Friedman, S. D., Howk, J. C., Chayer, P., et al. 2002, ApJS, 140, 37

Garcia Lopez, R. J., Lambert, D. L., Edvardsson, B., et al. 1998, ApJ, 500,241

Garcia Lopez, R. J., Israelian, G., Rebolo, R., et al. 2001, NewAR, 45, 519

Gibson, B. K., Penton, S. V., Giroux, M. L., et al. 1999, Bull. AAS, 31,1476

Gibson, B. K., Giroux, M. L., Penton, S. V., et al. 2001, AJ, 122, 3280

Gilmore, G., Gustaffson, B., Edwardsson, B., \& Nissen, P. 1992, Nature, 357, 379

Gilmore, G., Wyse, R. F. G., \& Jones, J. B. 1995, AJ, 109, 1095

Grevesse, N., \& Sauval, A. J. 1998, Space Sci. Rev., 85, 16

Gunn, J. E., \& Gott, J. R. III 1972, ApJ, 176, 1

Hébrard, G., Lemoine, M., Vidal-Madjar, A., et al. 2002, ApJS, 140, 103

Hedrick, D., \& Cox, D. P. 1977, ApJ, 215, 208

Howk, J. C., Sembach, K. R., \& Savage, B. D. 2000, ApJ, 543, 278

Hunt, R., \& Sciama, D. W. 1972, MNRAS, 157, 335

Israelian, G., Garcia-Lopez, R., \& Rebolo, R. 1998, ApJ, 507, 805

Jacq, T., Baundry, A., Walmsley, C. M., \& Caselli, P. 1999, A\&A, 347, 957

Jiang, I.-G., \& Binney, J. 1999, MNRAS, 303, L7

Jorgensen, B. R. 2000, A\&A, 363, 947

Josey, S. A., \& Tayler, R. J. 1991, MNRAS, 251, 474

Josey, S. A., \& Arimoto, N. 1992, A\&A, 255, 105

Kahn, F. D., \& Woltjer, L. 1959, ApJ, 130, 705

Knight, P. A., \& Ponman, T. J. 1997, MNRAS, 289, 955

Kohl, J. L., Parkinson, W. H., \& Withbroe, G. L. 1977, ApJ, 212, L101

Kormendy, J. 1979, ApJ, 227, 714

Kotoneva, E., Flynn, C., Chiappini, C., \& Matteucci, F. 2002, MNRAS, 336, 879

Kruk, J. W., Howk, J. C., André, M., et al. 2002, ApJS, 140, 19

Larson, R. B. 1972a, Nature, 236, 21

Larson, R. B. 1972b, Nat. Phys. Sci., 236, 7

Larson, R. B. 1976, MNRAS, 176, 31

Lehner, N., Keenan, F. P., \& Sembach, K. R. 2001, MNRAS, 323, 904

Lehner, N., Gry, C., Sembach, K. R., et al. 2002, ApJS, 140, 81

Lemke, M., Lambert, D. L., \& Edvardsson, B. 1993, PASP, 105, 468

Lemoine, M., Vidal-Madjar, A., Hébrard, G., et al. 2002, ApJS, 140, 67

Lepine, J. R. D., \& Duvert, G. 1994, A\&A, 286, 60

Lopez-Corredoira, M., Beckman, J. E., \& Casuso, E. 1999, A\&A, 351, 920

Lubowich, D. A., Pasachoff, J. M., Balonek, T. M., et al. 2000, Nature, 405,1025

Lynden-Bell, D. 1975, Vistas Astron., 19, 299

Mashonkina, L., Gehren, T., Travaglio, C., \& Borkova, T. 2003, A\&A, 397,275

Mayor, M., \& Vigroux, L. 1981, A\&A, 98, 1

Meusinger, H., Reimann, H., \& Stecklum, B. 1991, A\&A, 245, 51

Meyer, D. M., Jura, M., Hawkins, I., \& Cardelli, J. A. 1994, ApJ, 437, L59

Mirabel, I. F. 1982, ApJ, 256, 112

Moos, H. W., Sembach, K. R., Vidal-Madjar, A., et al. 2002, ApJS, 140,3

Muller, C. A., Oort, J. H., \& Raimond, E. 1963, C. R. Acad. Sci. Paris, 257, 1661

Murali, C., Katz, N., Hernquist, L., Weinberg, D. H., \& Dave, R. 2002, ApJ, 571, 1

Naoumov, S. O. 1999, Ph.D. Thesis, Univ. of North Carolina at Chapel Hill

Nissen, P., Gustafsson, B., Edvardsson, B., \& Gilmore, G. 1994, A\&A, 285,440
Olivier, S. S., Primack, J. R., \& Blumenthal, G. R. 1991, MNRAS, 252, 102

Oort, J. H. 1966, Bull. Astron. Inst. Netherlands, 18, 421

Oort, J. H. 1970, A\&A, 7, 381

Pagel, B. E. J., \& Patchett, B. E. 1975, MNRAS, 172, 13

Pagel, B. E. J. 1987, in The Galaxy, ed. G. Gilmore, \& B. Carswell (D. Reidel), 341

Pagel, B. E. J. 1997, in Nucleosynthesis and Chemical Evolution of Galaxies (Cambridge: C.U.P.)

Pardi, M. Ch., Ferrini, F., \& Matteucci, F. 1995, ApJ, 444, 207

Pettini, M., \& Bowen, D. A. 2001, ApJ, 560, 41

Phillipps, S. 1993, MNRAS, 263, 86

Phookun, B., Vogel, S. N., \& Mundy, L. G. 1993, ApJ, 418, 113

Pilyugin, L. S., \& Edmunds, M. G. 1996, A\&A, 313, 783

Piskunov, N., Wood, B. E., Linsky, J. L., Dempsey, R. C., \& Ayres, T. R. 1997, ApJ, 474, 31

Pitts, E., \& Tayler, R. J. 1996, MNRAS, 280, 1101

Polehampton, E. T., Baluteau, J. P., Ceccarelli, C., Swinyard, B. M., \& Caux, E. 2002, A\&A, 388, L44

Prantzos, N., Casse, M., \& Vangioni-Flam, E. 1993, ApJ, 403, 630

Primas, F., Duncan, D. K., Peterson, R. C., \& Thorburn, J. A. 1999, A\&A, 343, 545

Primas, F., Asplund, M., Nissen, P. E., \& Hill, V. 2000a, A\&A, 364, L42

Primas, F., Molaro, P., Bonifacio, P., \& Hill, V. 2000b, A\&A, 362, 666

Prochaska, J. X., Naumov, S. O., Carney, B. W., McWilliam, A., \& Wolfe, A. M. 2000, AJ, 120, 2513

Pryor, C., \& Leccar, M. 1983, ApJ, 269, 513

Putman, M. E., de Heij, V., Staveley-Smith, L., et al. 2002, AJ, 123, 873

Ramaty, R., Scully, S. T., Lingenfelter, R. E., \& Kozlovsky, B. 2000, ApJ, 534, 747

Rebolo, R., Garcia-Lopez, R. J., \& Perez del Taoro, M. R. 1995, in The Light Element Abundances, ed. P. Crane (Springer), 420

Robin, A. C., Haywood, M., Creze, M., Ojha, D. K., \& Bienayme, O. 1996, A\&A, 305, 125

Rocha-Pinto, H. J., \& Maciel, W. J. 1996, MNRAS, 279, 447

Rocha-Pinto, H. J., Scalo, J., Maciel, W. J., \& Flynn, C. 2000, A\&A, 358,869

Ryan, S., Norris, J., Bessell, M., \& Deliyannis, C. 1992, ApJ, 388, 184

Ryden, B. S. 1988, ApJ, 329, 589

Sarajedini, A., \& van Duyne, J. 2001, AJ, 122, 2444

Savage, B. D., Sembach, K. R., Wakker, B. P., et al. 2003, ApJS, 146, 125

Sembach, K. R., Savage, B. D., Lu, L., \& Murphy, E. M. 1999, ApJ, 515, 108

Sembach, K. R., Wakker, B. P., Savage, B. D., et al. 2003, ApJS, 146, 165

Schmidt, M. 1963, ApJ, 137, 758

Songaila, A., Cowie, L. L., \& Weaver, H. 1988, ApJ, 329, 580

Sonneborn, G., André, M., Oliveira, C., et al. 2002, ApJS, 140, 51

Takeuchi, T. T., \& Hirashita, H. 2000, ApJ, 540, 217

Tantalo, R., Chiosi, C., Bressan, A., Marigo, P., \& Portinari, L. 1998, A\&A, 335, 823

Thon, R., \& Meusinger, H. 1998, A\&A, 338, 413

Tinsley, B. 1977, ApJ, 216, 548

Tinsley, B. M. 1980, Fund. Cosmic. Phys., 5, 287

Tosi, M. 1988, A\&A, 197, 47

Toth, G., \& Ostriker, J. P. 1992, ApJ, 389, 5

Tripp, T. M., Wakker, B. P., Jenkins, E. B., et al. 2003, AJ, 125, 3122

Tufte, S. L., Reynolds, R. J., \& Haffner, L. M. 1998, ApJ, 504, 773

Twarog, B. A. 1986, ApJ, 242, 242 
Valle, G., Ferrini, F., Galli, D., \& Shore, S. N. 2002, ApJ, 566, 252 van den Bergh, S. 1962, AJ, 67, 486

Vangioni-Flam, E., Casse, M., Audouze, J., \& Oberto, Y. 1990, ApJ, 364,586

Vaughan, A. H., \& Preston, G. W. 1980, PASP, 92, 385

Verschuur, G. L. 1969, ApJ, 156, 771

Wakker, B. P., \& Schwarz, U. J. 1991, A\&A, 250, 484

Wakker, B. P. 1991, A\&A, 250, 499

Wakker, B. P., \& van Woerden, H. 1991, A\&A, 250, 509

Wakker, B. P., \& van Woerden, H. 1997, ARA\&A, 35, 217

Wakker, B. P., Howk, J. C., \& Savage, B. D. 1999a, in Stromlo Workshop on High-Velocity Clouds, ed. B. K. Gibson, \& M. E. Putman, ASP Conf. Ser., 166, 26
Wakker, B. P., van Woerden, H., \& Gibson, B. K. 1999b, in Stromlo Workshop on High-Velocity Clouds, ed. B. K. Gibson, \& M. E. Putman, ASP Conf. Ser., 166, 311

Wakker, B. P., Howk, J. C., Savage, B. D., et al. 1999, Bull. AAS, 31, 887

Wakker, B. P. 2001, ApJS, 136, 463

Wakker, B. P., Savage, B. D., Sembach, K. R., et al. 2003, ApJS, 146, 1 Weiner, B. J., Vogel, S. N., \& Williams, T. B. 1999, AAS, 195, 9703

Wood, B. E., Linsky, J. L., Hébrard, G., et al. 2002, ApJS, 140, 91

Worthey, G., Dorman, B., \& Jones, L. A. 1996, AJ, 112, 948

Wyse, R. F. G., \& Gilmore, G. 1995, AJ, 110, 2771 